Influence of TMF-induced fluid flow on the microstructure of AI-Si7-Mn1 wt.\% alloy

This article has been downloaded from IOPscience. Please scroll down to see the full text article.

2012 IOP Conf. Ser.: Mater. Sci. Eng. 27012044

(http://iopscience.iop.org/1757-899X/27/1/012044)

View the table of contents for this issue, or go to the journal homepage for more

Download details:

IP Address: 129.247.247.240

The article was downloaded on 12/01/2012 at 14:54

Please note that terms and conditions apply. 


\title{
Influence of TMF-induced fluid flow on the microstructure of Al-Si7-Mn1 wt.\% alloy
}

\author{
A Orth and L Ratke \\ Institut für Materialphysik im Weltraum, Deutsches Zentrum für Luft- und Raumfahrt, Linder \\ Höhe, 51147 Köln \\ E-mail: Andreas.Orth@DLR.de
}

\begin{abstract}
The precipitation of Mn-rich intermetallics in AlSiMn alloys during solidification ahead of the mushy zone affects the solidification microstructure, especially if fluid flow is present. Recently Steinbach and Ratke reported a barrier effect of $\alpha$-AlMnSi, meaning these intermetallics prevent fluid flow to enter the mush. To investigate this effect further we studied the solidification of AlSi7Mn1 with a fluid flow field induced by a traveling magnetic field (TMF). Samples were molten and directionally solidified within a silica aerogel crucible at various constant solidification velocities between 0.03 and $0.24 \mathrm{~mm} / \mathrm{s}$. The application of two separate heaters allowed the fixation of constant temperature gradients in the solid and liquid parts of the samples, the use of a transparent aerogel as crucible material permitted direct optical verification of the desired solidification velocity using an infrared line camera. Three collinear coils induced a TMF of approximately $5 \mathrm{mT}$ strength, traveling either up or downward in the direction of the sample axis. The microstructures of the processed samples were studied using light microscopy and SEM-EDX and characterised by the primary and secondary dendrite arm spacing, the distribution of intermetallic phases as well as the radial segregation of primary phase and eutectic. Results are presented which show differences between samples with and without TMF-induced fluid flow. We noticed a radial macro-segregation dependent on the orientation of the TMF and the effects of the induced fluid flow on the primary and secondary dendrite arm spacing are examined.
\end{abstract}

\section{Introduction}

In a world of increasing environmental awareness, the recyclability of scrap metals such as aluminum is becoming increasingly important. A common impurity in secondary aluminum is manganese. When casting a commercial aluminum alloy, small concentrations of Mn can already lead to a change in the microstructure. The low solubility of Mn in an Al-matrix leads to the formation of intermetallic phases (Mn-rich aluminides) of different size and morphology, which in turn influences the mechanical properties of the workpiece.

On the one hand, removement of these impurities is elaborate and costly. On the other hand, fluid flow is known to also have a great influence on the microstructure formation. Fluid flow is always present when casting in a mould, either through natural convection or forced by the casting process itself. Another possibility is the deliberate induction of fluid flow in the melt by means of changing magnetic fields.

The goal of our research is to gain a better understanding of the effect of fluid flow on the formation and resulting morphology of the intermetallic phases. This could lead to a cost efficient method for foundries to control the impurity distribution, rather than having to remove them. 


\section{Experimental}

The processed samples (Al-Si7-Mn1 wt-\%) were produced from pure components: $\mathrm{Al}$ (99.999\% Hydro Aluminium Deutschland $\mathrm{GmbH}$ ), Si of spectral purity (Crystal Growth Laboratory Berlin, Germany) and Mn (99,7\% Haines \& Maassen Metallhandelsges.mbH.) were machined to cylindrical form of $8 \mathrm{~mm}$ diameter and $120 \mathrm{~mm}$ length.

The furnace assembly consists of three parts: at the top and bottom are two heaters, each individually controlled to enable upward directional solidification with a constant solidification velocity. The two heaters are separated by an aerogel crucible, which creates an adiabatic solidification region.

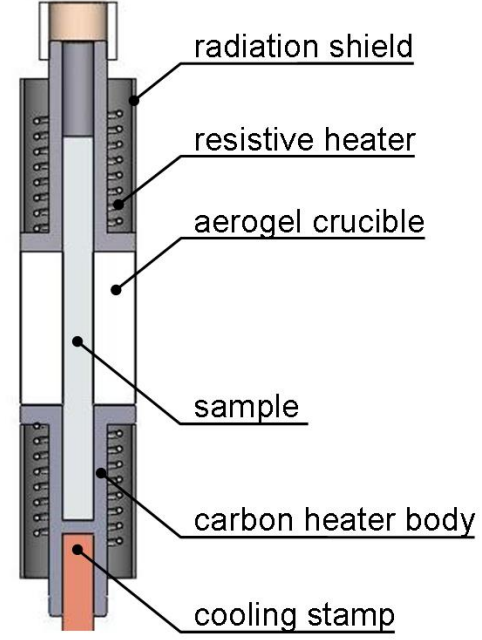

Figure 1. Cross section of the furnace assembly
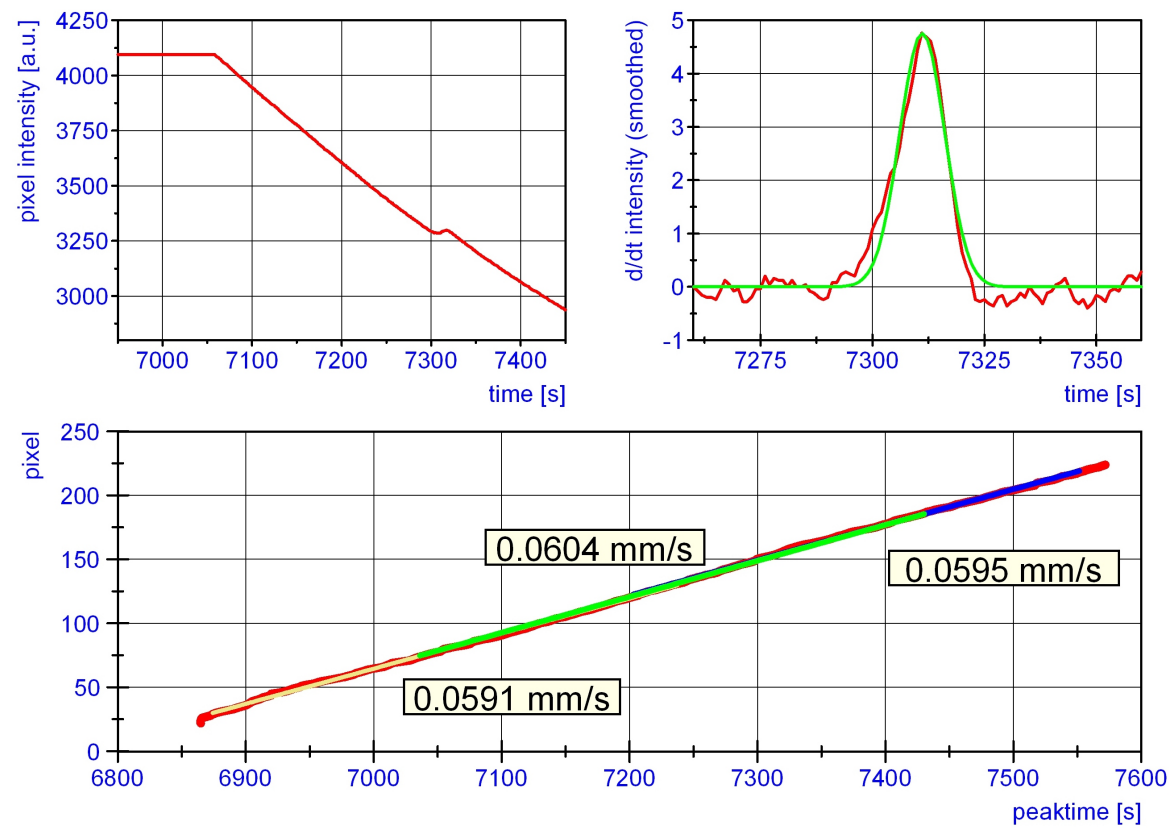

Figure 2. Intensity over time of one pixel (top left); smoothed derivative of intensity with fit peak (top right); peak times for all pixels, gauged from pix/s to $\mathrm{mm} / \mathrm{s}$ (bottom)
Each heater is made of a cylindrical carbon body around which an inconel coated, mineral insulated resistive heater is coiled. The coiled form facilitates the assembly, possible magnetic effects of the heater currents on the sample are shielded by the graphite, as was confirmed by measurements with a hall sensor. The heaters are enclosed by a thin steel tube, the free spacing is filled with graphite glue (931 Graphite, Cotronics Corporation Brooklyn, N.Y., USA) for an optimal thermal contact. In addition, a water cooled copper stamp is inserted into the bottom heater for higher cooling velocities, as can be seen in figure 1 .

The aerogel crucible is optically transparent, which enables an observation of the solidification front with an infrared line camera. The transition from liquid to solid state causes a jump in the local emissivity of the melt which is recorded and thus allows for online measurement of the solidification velocity, see figure 2 .

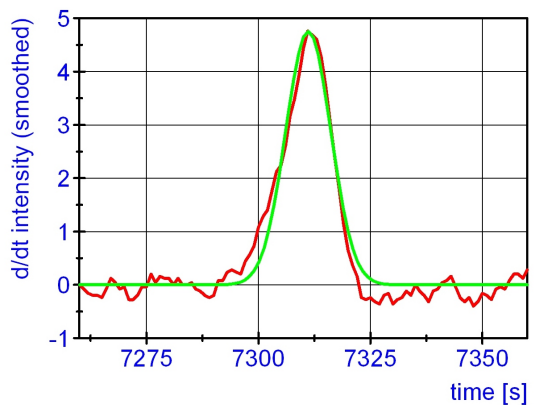


Together with a constant temperature decline in the heaters, a constant temperature gradient in both the liquid and solid parts of the sample is ensured, as per

$$
G_{L / S}=\frac{\dot{T}_{L / S}}{v}
$$

Three collinear coils are mounted on a heat sink which encloses the entire furnace setup. The voltage applied to each coil can be separately adjusted by a rotary current transformer (operating at $50 \mathrm{~Hz}$ ) from 0 to $250 \mathrm{~V}$. The phase difference between two adjacent coils is set to $\pm 60^{\circ}$ for all coils, resulting in a traveling magnetic field (TMF), moving either up or downward along the sample axis. The coil bearings were built from hard paper and the heat sink slotted to minimise inductive shielding of the sample by the surrounding setup. The target currents for each coil were determined with a hall sensor such that the field strength of the TMF was nearly constant throughout the sample volume, set to $5 \mathrm{mT}$. Great care was taken to monitor and maintain these currents during sample processing, as the radiation from the furnace and the self-heating of the coils would increase their electrical resistance and thus dampen the field.

An aluminized rectangular prism is fit in between the furnace and the TMF heat sink to deflect the optical path for the infrared solidification measurement, as can bee seen in figure 3 . Finally, for increased thermal insulation, the entire setup is mounted inside a vacuum chamber.

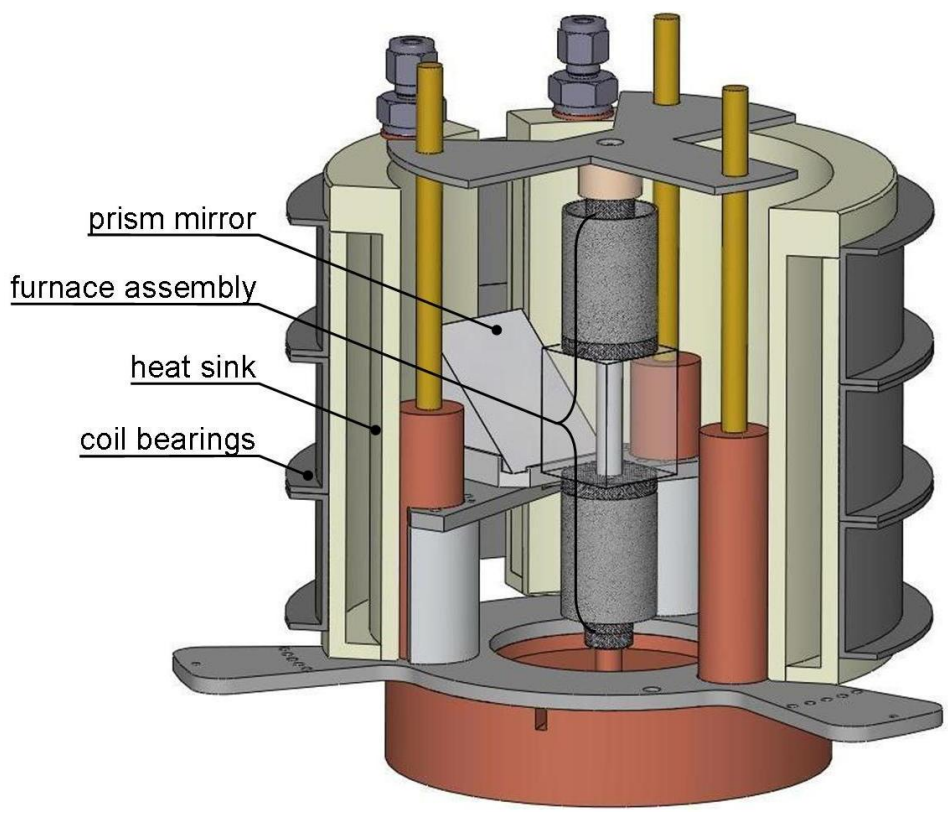

Figure 3. Furnace setup (from figure 1) and prism inside the TMF heat sink and coil bearings. The camera (not shown) is mounted above the setup, looking down onto the prism.

\section{Results and discussion}

Samples were processed with five solidification velocities $(0.03,0.045,0.06,0.12$ and $0.24 \mathrm{~mm} / \mathrm{s})$ and either upward, downward or no TMF. The samples were then cut into selected slices (see figure 4), micro-milled, polished and examined under an optical microscope. From the longitudinal cross sections, the secondary dendrite arm spacing (SDAS) was obtained, and from the transversal cross sections the primary dendrite arm spacing (PDAS) as well as the radial segregation of primary phase and eutectic. For this last measurement, the surface area of both phases was calculated using image processing software. The microscope image was converted to greyscale and the two phases separated by a threshold value. As this value depends on brightness and contrast, it was assigned manually for each picture. Therefore, the absolut values of these 


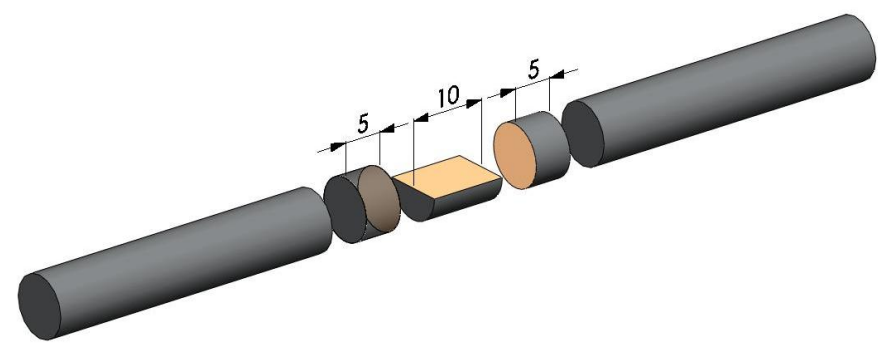

Figure 4. Positions of the examined transversal and longitudinal cross sections (in $\mathrm{mm}$ )
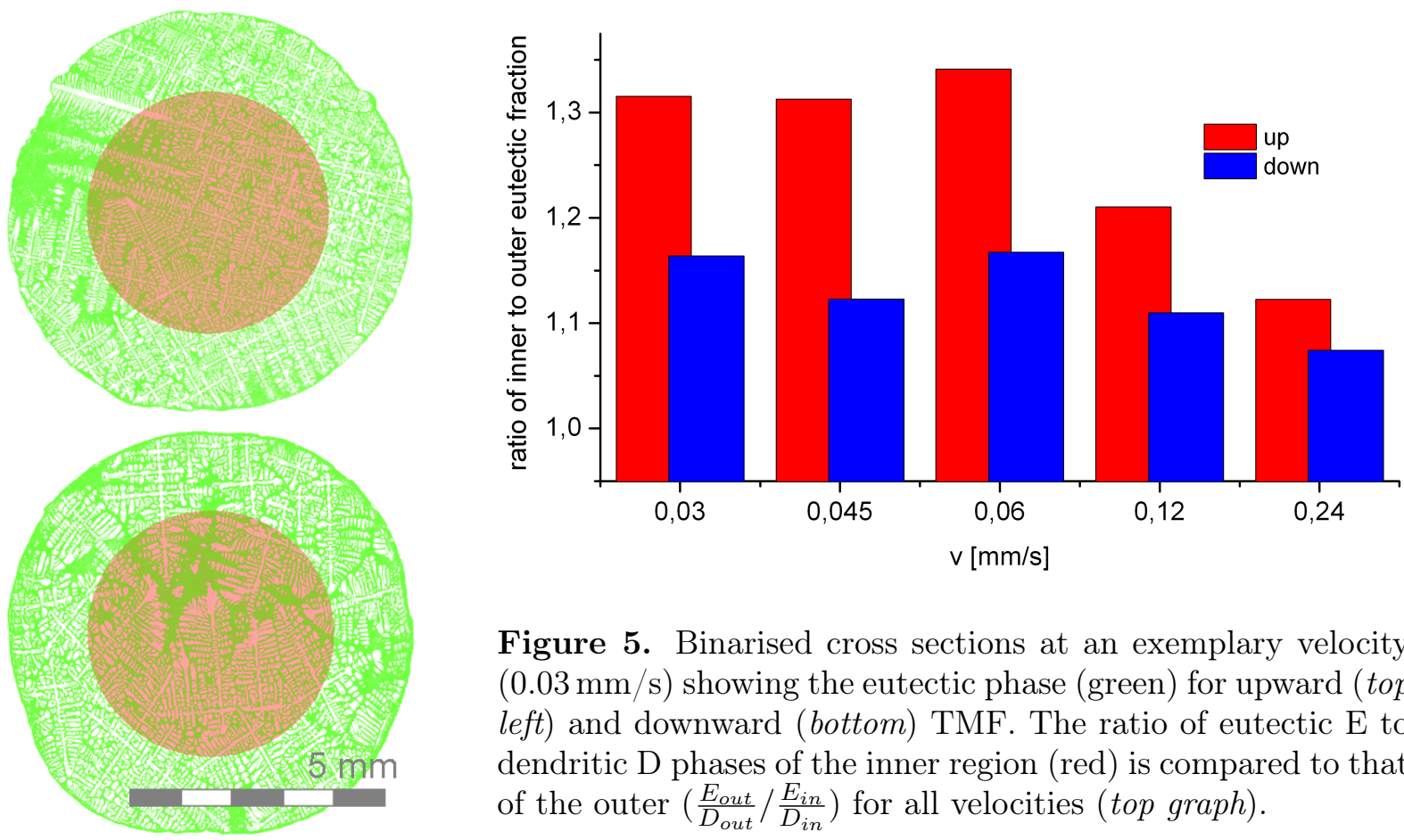

Figure 5. Binarised cross sections at an exemplary velocity $(0.03 \mathrm{~mm} / \mathrm{s})$ showing the eutectic phase (green) for upward (top left) and downward (bottom) TMF. The ratio of eutectic E to dendritic D phases of the inner region (red) is compared to that of the outer $\left(\frac{E_{\text {out }}}{D_{\text {out }}} / \frac{E_{\text {in }}}{D_{\text {in }}}\right)$ for all velocities (top graph).

measurements are subject to fluctuations and not readily comparable to one another. Thus the eutectic fraction was calculated as the area of the eutectic phase divided by the area of the primary phase dendrites, and then the ratio of this value was taken for the inner and outer regions of the cross section. The value of this fraction was found to be rather invariant under small changes in the above mentioned threshold value. The border between inner and outer region was chosen at $d=0.6 \cdot d_{\text {sample }}$, for this is where the flow is expected to change orientation, according to numerical simulations [1]. Two transversal cross sections were examined per sample, and since the results from these were almost equal, the respective mean values were considered for further analysis.

Figure 5 shows this calculation for one exemplary solidification velocity as well as the results for all examined velocities. As to be expected, the segregation decreases with increasing solidification velocity, for both TMF orientations. However there seems to be a sort of plateau for velocities lower than $0.06 \mathrm{~mm} / \mathrm{s}$.

Depending on the orientation of the TMF, the flow in the molten sample will be either upward in the middle, towards the outside at the top, down along the outer surface and back inward at the solid liquid interface, or vice versa [2]. The radial flow at the interface towards either the sample middle or the circumference will lead to an accumulation of the heavier atoms in the melt in that region and thus a larger portion of eutectic lamella being solidified there.

The PDAS is measured directly between neighbouring dendrites within one grain. Figure 
6 shows the values obtained for the different velocities (error bars indicating the standard deviation). There is no apparent effect of the traveling magnetic field on the PDAS, in contrast to past experiments using rotating magnetic fields [3]. Further, a transition from dendritic to columnar growth can be observed for decreasing velocities. This coincides with the above mentioned plateau for the eutectic fraction.

Different models for the calculation of the PDAS can be found in the literature [4], [5], [6], [7], all coinciding in the prediction that an increase in the solidification velocity will lead to a decrease of the dendrite spacing. As figure 6 shows, we have too little data points at this time to perform a significant analytical fit. More cross sections of the already processed samples should be examined (to reduce the statistical error), as well as further samples solidified at additional velocities.

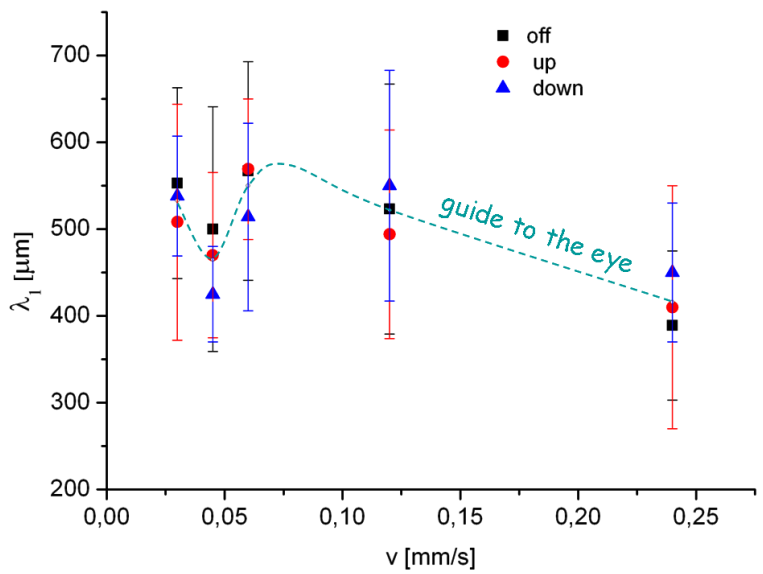

Figure 6. Primary DAS for different velocities. The transition from columnar to dendritic growth is clearly recognisable.

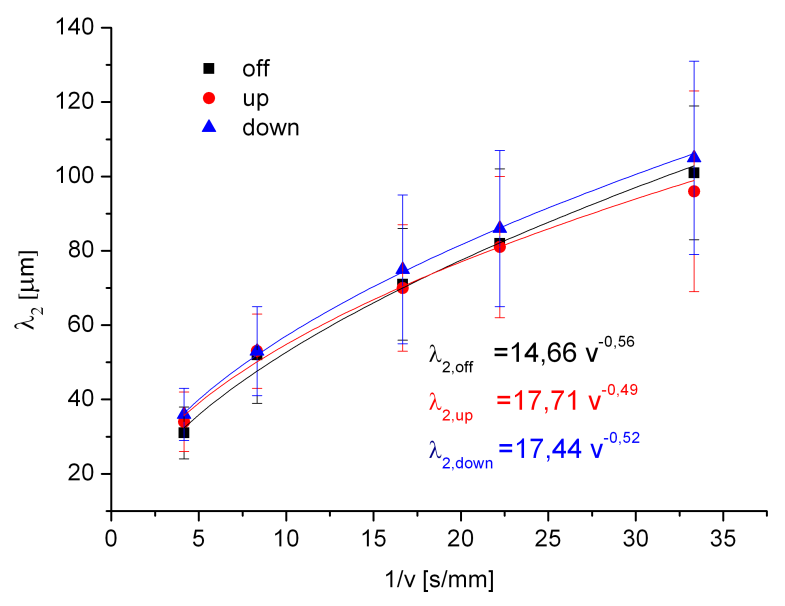

Figure 7. Secondary DAS shows no apparent effect of the TMF, while the velocity dependence seems to follow a $\lambda_{2} \propto v^{-1 / 2}$ law.

The SDAS is obtained from the longitudinal cross sections and can be seen in figure 7 . There is no visible effect of the TMF on the SDAS, all three curves seem to follow the coarsening law. Slower solidification velocities lead to longer solidification times, allowing more time for mass transport between two neighboring secondary dendrite arms [8]:

$$
\lambda_{2} \propto \frac{1}{v^{b}} \propto t_{f}^{b}
$$

The coarsening exponent $b$ is expected to be $1 / 3$ for purely diffusive mass transport and should grow to $1 / 2$ for forced flow [9]. As can be seen in figure 7 , we obtain an exponent of $1 / 2$ for all three curves, including the case of no TMF. So far we have found no explanation for this enhanced coarsening effect.

Further measurements of the already processed samples as well as additional ones at other solidification velocities are needed to obtain reliable conclusions. Further, comparative measurements with binary Al-Si7 are underway, with which we hope to gain more insight into the possible cause for this apparent additional coarsening.

Finally, the distribution of the intermetallic phases was investigated using SEM-EDX. Figure 8 shows these intermetallics within the sample. Although the manganese rich phase is expected to form first, it does not act as a nucleation site for the following, aluminum rich dendritic phase. All of the segregated, Mn-rich phase is found in the interdendritic region. A detailed examination of the effects of the TMF on this distribution will follow in the near future. 


\section{Summary}

Samples of Al-Si7-Mn1 wt.\% were directionally solidified at various solidification velocities while being exposed to a travelling magnetic field, leading to forced fluid flow in the melt. Our results show that macrosegregation of Si-rich phases can be achieved either in the middle or towards the outer rim of the sample, depending on the orientation of the TMF. However, the presence of the magnetic field has shown no measurable influence on the primary or secondary arm spacing of the dendrites. Further research with samples at additional velocities and purely binary Al-Si7 wt.\% composition is ongoing.

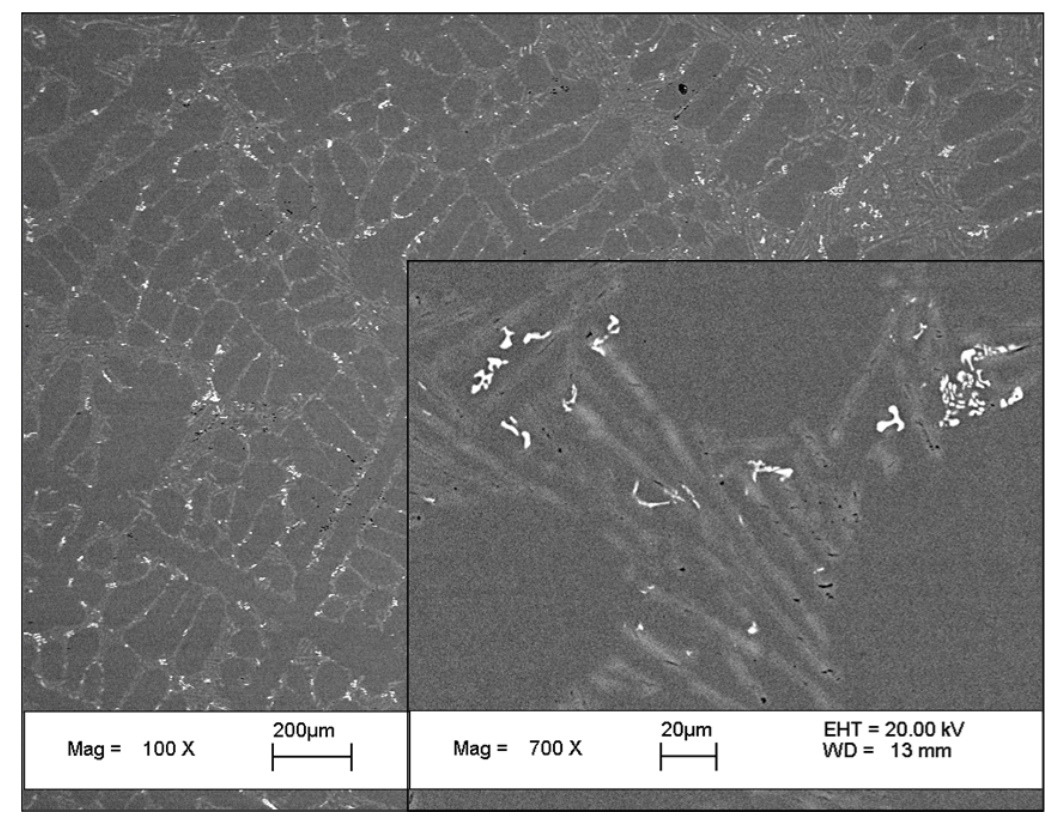

Figure 8. SEM image of a transversal cross section, the dendritic structure is well visible. EDX analysis identified the Mn-rich precipitates as the bright white areas.

\section{References}

[1] Grants I and Gerbeth G 2004 J. Crystal Growth 269 pp 630-8

[2] Lantzsch R et al 2007 J. Crystal Growth 305 pp 249-56

[3] Steinbach S and Ratke L 2009 Int. J. Cast Met. Res. 22 pp 290-3

[4] Hunt J D 1979 Solidification and Casting of Metals (London: The Metals Society) 192 p 3

[5] Fischer D J and Kurz W 1981 Acta Metall. 29 pp 11-20

[6] Trivedi R 1984 Metall. Trans. A A15 pp 977-82

[7] Sahm P R and Ma D 1992 Acta Metall. 40 pp 251-7

[8] Kurz W and Fisher D J 1989 Fundamentals of Solidification (Switzerland: Trans. Tech. Publications)

[9] Steinbach S et al 2007 Trans. Ind. Inst. Met. 2-3 60 pp 137-41 